\title{
Histoplasmosis felina: reporte de un caso fatal
}

\author{
Sierra, C.A. ${ }^{1}$; Martínez, L.C. ${ }^{1}$; Forero, F.M. ${ }^{2}$ \\ ${ }^{1}$ Grupo de Investigación, Universidad de Santander, Campus Valledupar, Colombia. \\ ${ }^{2}$ Universidad Nacional de Colombia, Facultad de Medicina Veterinaria y Zootecnia. \\ E-mail: ca.sierra@mail.udes.edu.co
}

\begin{abstract}
Resumen
Sierra, C.A.; Martínez, L.C.; Forero, F.M.: Histoplasmosis felina: reporte de un caso fatal. Rev. Vet. 31: 2, 192-195, 2020. Se describe el caso de una gata mestiza de 4 meses de edad, encontrada en situación de abandono. Al examen clínico presentaba circunstancias poco favorables: baja condición corporal, pelo hirsuto y sintomatología respiratoria con secreciones nasales y oculares bilaterales. Las constantes fisiológicas mostraron taquicardia, disnea y fiebre; a la auscultación se percibieron estertores, estridores, sibilancias y aumento de la matidez pulmonar. Los resultados del hemograma evidenciaron alteraciones asociadas a una anemia regenerativa, anisocitosis y policromatofilia moderadas, desvío a la izquierda con presencia de bandas, trombocitopenia e inclusiones intracitoplasmáticas compatibles con Histoplasma $s p$. Los exámenes radiológicos demostraron un patrón intersticial característico de enfermedad pulmonar. Se efectuó tratamiento con oxitetraciclina IV $(10 \mathrm{mg} / \mathrm{kg} \mathrm{c} / 12 \mathrm{~h})$ y ketoconazol PO $(10 \mathrm{mg} / \mathrm{kg} \mathrm{c} / 24 \mathrm{~h})$. También se aplicó terapia sintomática de sostenimiento, la cual fue insuficiente para conservar la vida del animal. La necropsia y consecuente histopatología de pulmón, corazón, médula ósea, bazo, hígado, riñón e intestino, confirmaron el diagnóstico de histoplasmosis. Las lesiones observadas microscópicamente permiten sugerir un proceso infeccioso multifactorial; primero una enfermedad neoplásica (linfoma multicéntrico difuso de células grandes en médula ósea y linfonodo), asociada a virus de la leucemia felina, negativa inicialmente por inmunocromatografía y luego micosis sistémica (especialmente pulmón y linfonodo) ocasionada por Histoplasma sp.
\end{abstract}

Palabras clave: gato, histoplasmosis, síntomas, lesiones, rayos X, análisis.

\begin{abstract}
Sierra, C.A.; Martínez, L.C.; Forero, F.M.: Feline histoplasmosis: report of a fatal case. Rev. Vet. 31: 2, 192-195, 2020. A 4-month-old mixed-breed abandoned was submitted to a veterinary hospital for clinical examination. The patient presented poor conditions: low body condition, shaggy hair, and respiratory symptoms with ocular and bilateral nasal secretions. The physiological parameters showed tachycardia, dyspnea and fever; on auscultation, rales, stridor, wheezing and increased pulmonary dullness were noted. The results of complete blood count showed alterations associated with a regenerative anemia; moderate anisocy tosis and polychromatophilia; deviation to the left with presence of bands and thrombocy topenia; intracytoplasmic inclusions compatible with Histoplasma sp. Radiological examinations showed a characteristic interstitial pattern relative to lung disease. Treatment was started with oxytetracycline IV $(10 \mathrm{mg} / \mathrm{kg} / 12 \mathrm{~h})$ and ketoconazole PO $(10 \mathrm{mg} / \mathrm{kg} / 24 \mathrm{~h})$, the most prescribed antifungal for these infections, as well as symptomatic supportive therapy. Unfortunately, the patient died despite all the efforts. Necropsy was performed. The presence of granulomas together with the histopathological findings confirmed the diagnose of histoplasmosis. The lesions observed microscopically suggest a multifactorial infectious process: first, a neoplastic disease (diffuse large cell lymphoma in bone marrow and lymph node) associated with feline leukemia virus, followed by systemic mycosis (especially in lungs and lymph nodes) caused by Histoplasma sp.
\end{abstract}

Key words: cat, histoplasmosis, symptoms, lesions, X-rays, analysis.

\section{INTRODUCCIÓN}

La histoplasmosis o mal de Darling es una micosis sistémica que se presenta tanto en seres humanos como

Recibido: mayo 2020 / Aceptado: agosto 2020 en animales domésticos de interés veterinario ${ }^{1,7}$. Se ha descrito como la segunda micosis sistémica más común en gatos, los cuales muestran mayor susceptibilidad que los perros. Geográficamente está relacionada con áreas tropicales y subtropicales y se cree que tiene distribución mundial, salvo en el continente antártico. 
Estas micosis usualmente no representan una afección importante a nivel epidemiológico, ya que la condición inicial para que logren generar un impacto en el huésped es la inmunosupresión, o la entrada traumática del hongo en una lesión. La principal vía de infección parece ser la inhalación de conidios procedentes del ambiente ${ }^{2}$.

Histoplasma $s p$ es un hongo dimórfico que crece en fase de levadura en tejidos y cultivos incubados a $37^{\circ} \mathrm{C}$. Su fase de moho prospera en cultivos incubados entre 22 y $25^{\circ} \mathrm{C}$ y como saprofito en la tierra ${ }^{5}$.

Existen tres especies de Histoplasma (capsulatum, duboisii y farciminosum), que varían en su distribución geográfica y su virulencia. Las formas de levadura son pequeñas para los hongos ( 2 a $4 \mu \mathrm{m}$ ) y se reproducen por gemación (blastoconidias). Los micelios son tabicados y producen microconidias; la estructura diagnóstica se denomina macroconidia tuberculada porque tiene proyecciones digitiformes, radiales y de pared gruesa ${ }^{5}$.

La designación de H. capsulatum es en realidad inadecuada, porque no forma cápsulas. Proviene de los halos que se observan alrededor de las levaduras en cortes hísticos, lo cual es causado por un artefacto en los métodos histopatológicos habituales ${ }^{3}$.

Luego de la inhalación, dichos conidios prosiguen desde la fase micelar a la de levadura, la cual será fagocitada por las células especializadas del sistema inmunológico del huésped. Una vez allí se replican y luego se diseminan por los sistemas hemático y linfático, dando lugar a una infección sistémica ${ }^{6}$.

Los casos reportados para lesiones sistémicas suelen alcanzar compromisos orgánicos mayores, que involucran órganos importantes y que -en algún momentopueden ser comprometedores para la vida del animal ${ }^{4,5}$.

En este reporte se describe un caso clínico relacionado con un felino proveniente de la zona de los llanos orientales de Colombia, que fue atendido en una clínica privada de la ciudad de Bogotá. El paciente se encontraba en condiciones de abandono y presentaba un cuadro de desnutrición crónica acompañado de una deteriorada condición corporal, con alteraciones de las faneras cutáneas y un problema respiratorio bastante evidente, con rales audibles sin la necesidad de utilizar fonendoscopio.

\section{MATERIAL Y MÉTODOS}

Examen clínico. Una hembra felina, mestiza, de 4 meses de edad, presentó síntomas como baja condición corporal, pelo hirsuto y sintomatología respiratoria con secreciones nasales y oculares bilaterales. Las constantes fisiológicas mostraron taquicardia, disnea y fiebre; a la auscultación se percibieron estertores, estridores, sibilancias y aumento de la matidez pulmonar.

El animal presentaba desnutrición y retraso en su tasa normal de crecimiento, con deshidratación importante $(8 \%)$, un cuadro respiratorio con disnea, secreción nasal mucopurulenta, estridores, sibilancias y sonidos compatibles con matidez pulmonar, así como consolidación de las zonas craneal y caudal de los pulmones, a nivel bilateral.

También presentó una conjuntivitis bilateral con secreción purulenta, blefaroespasmo y fotofobia, además de una dermatitis multifocal con hiperqueratosis. Dentro de los diagnósticos diferenciales se incluyó: neumonía infecciosa, laringotraqueitis infecciosa felina, calicivirus sp e infección por Mycoplasma haemofelis.

Frotis sanguíneo. Como muestra la Figura 1, el frotis sanguíneo evidenció la presencia de Histoplasma sp en células del sistema fagocitico mononuclear del felino.

Imagen radiográfica. En los aparatos respiratorio y digestivo (Figura 2), los rayos $\mathrm{X}$ evidenciaron un patrón intersticial a nivel de todo el parénquima pulmonar.

\section{RESULTADOS}

Pruebas de laboratorio. A1 ingresar el paciente a la consulta se procedió a extraer muestras de sangre para hemograma (Tabla 1), así como un raspado de piel. Se constató escasa concentración de hemoglobina y una activación de neutrófilos compatible con un cuadro inflamatorio agudo, corroborado por una marcada desviación a la izquierda y presencia de bandas. También se verificó disminución de la cantidad de plaquetas,

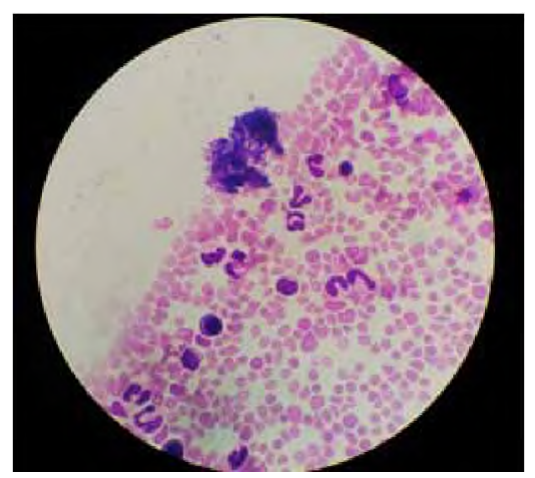

Figura 1. Frotis sanguíneo que evidencia la presencia de Histoplasma sp en células del sistema fagocitico mononuclear de la paciente.

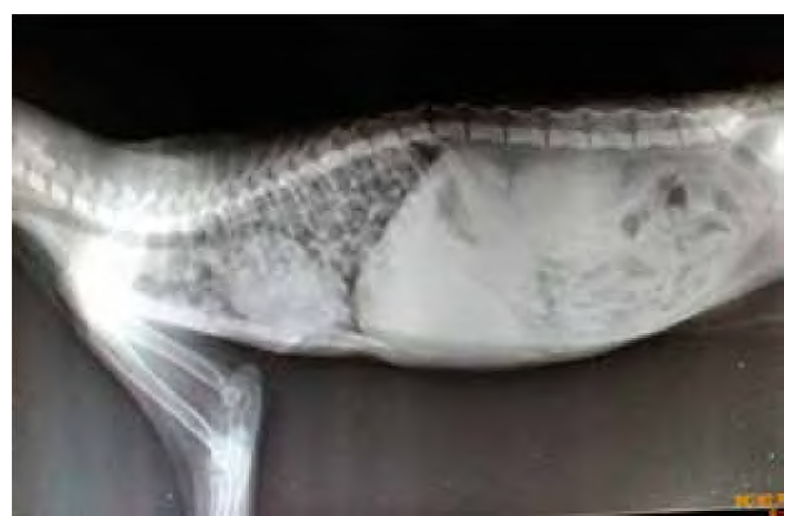

Figura 2. Radiografía en vista latero-lateral del paciente, exhibiendo un patrón intersticial a nivel de todo el parénquima pulmonar. 
Tabla 1. Análisis hematológico del paciente.

\begin{tabular}{|c|c|c|}
\hline parámetro & valor obtenido & VDR \\
\hline eritrocitos $\times 10^{12} / \mathrm{L}$ & 4,46 & $4,6-10$ \\
\hline hemoglobina $g / L$ & 57 & $93-153$ \\
\hline hematocrito \% & 16,0 & $28-49$ \\
\hline VCMfl & 35,0 & $39-52$ \\
\hline HCM pg & 12,7 & $13-21$ \\
\hline $\mathrm{CHCMg} / \mathrm{L}$ & 356 & $300-380$ \\
\hline plaquetas $\times 10^{3} / \mu \mathrm{L}$ & 132 & $300-800$ \\
\hline proteínas totales $\mathrm{g} / \mathrm{dL}$ & 7,2 & $5,7-7,9$ \\
\hline leucocitos $\times 10^{9} / \mathrm{L}$ & 10,5 & $5,5-19,5$ \\
\hline granulocitos NEB $\times 10^{9} / \mathrm{L}$ & 7,5 & $2,1-15$ \\
\hline linfocitos $\times 10^{9} / \mathrm{L}$ & 2,5 & $0,8-7,0$ \\
\hline monocitos $\times 10^{9} / \mathrm{L}$ & 0,5 & $0-1,9$ \\
\hline neutrófilos totales \% & $65 \%$ & $35-85$ \\
\hline neutrofilos en banda & $6 \%$ & - \\
\hline linfocitos $\%$ & $24 \%$ & $12-45$ \\
\hline monolitos \% & $5 \%$ & $2-9$ \\
\hline eosinofilos \% & $0 \%$ & $3-10$ \\
\hline basófilos \% & $0 \%$ & menor a 1 \\
\hline
\end{tabular}

VDR: valores de referencia. VCM: volumen corpuscular medio; HCM: hemoglobina corpuscular media; CHCM: concentración de hemoglobina corpuscular media; NEB: neutrófilos-eosinófilos-basófilos.

acompañada de una tendencia a la pancitopenia. El estudio incluyó la realización de un examen radiológico.

Daños en piel y tejidos. En las muestras de piel se encontraron alteraciones del crecimiento del pelo, constatándose un estadio catagénico y poca madurez y firmeza de sujeción en el folículo piloso. Adicionalmente se realizó un extendido para valorar de forma microscópica las características de las células sanguíneas, a través de muestras sometidas a la tinción de Gram. No se observaron estructuras compatibles con la presencia de hemoparásitos.

A su vez, los estudios realizados sobre los tejidos mostraron patrones de lesión tisular compatibles con una depleción metabólica apreciable, con un marcado compromiso tisular, que en síntesis complementaban el proceso de deterioro que mostraba el animal. Todas las alteraciones descritas en el reporte de histopatología demostraron un patrón de lesiones celulares más cercano a un fallo sistémico que vendría a ser consecuencia directa de la poca cantidad de eritrocitos que se encontraron circulando en el paciente.

Si bien es muy probable que la presencia del hongo que aquí se describe fuese determinante en el proceso que conllevó a la muerte del animal, las pobres condiciones generales en las que se encontraba el paciente coadyuvaron para su deceso.

Fisiopatología. La característica distintiva de la histoplasmosis es la infección de los ganglios linfáticos, bazo, médula ósea y otros elementos del sistema reticuloendotelial, con crecimiento intracelular en macrófagos fagocíticos. La infección inicial es de tipo pulmonar, a través de la inhalación de conidios infecciosos (esporas asexuales), los cuales se convierten en levaduras en el hospedador.

Ellos se unen a receptores de integrina o fibronectina y son captados con facilidad por "fagocitos profesionales". Las células dendríticas destruyen las levaduras invasoras, pero en el interior de los neutrófilos y macrófagos sobreviven a los efectos de la reacción oxidativa e inhiben la fusión de fagosoma-lisosoma.

Las características fundamentales en esta supervivencia y multiplicación, son la capacidad de Histoplasma capsulatum para fijar hierro y calcio del macrófago y para modular el $\mathrm{pH}$ del fagolisosoma ${ }^{4}$. $\mathrm{El} \mathrm{pH}$ ácido necesario para el efecto óptimo de destrucción en el lisosoma es elevado por $H$. capsulatum hacia un intervalo neutro, menos eficaz ( $\mathrm{pH} 6,0$ a 6,5).

Tratamiento. Se aplicó oxitetraciclina $(5 \mathrm{mg} / \mathrm{kg}$ cada $12 \mathrm{~h})$ y ketoconazol $(10 \mathrm{mg} / \mathrm{kg}$ cada $24 \mathrm{~h})$. También se efectuó terapia sintomática de sostenimiento, la cual fue insuficiente para conservar la vida del animal. Se realizó la necropsia del paciente para efectuar el diagnóstico histopatológico en distintos órganos.

\section{DISCUSIÓN}

La histopatología reportada por el laboratorio confirmó el resultado definido con anterioridad en la placa del frotis sanguíneo inicial, completando los exámenes que se tomaron antes del deceso de la paciente, incluida la imagenología. Como aseguran los investigadores, los signos clínicos de estos casos son bastante frecuentes en la histoplasmosis felina ${ }^{6}$.

Además, existía una fuerte presencia sistémica, situación importante sobre alguna patología preexistente, reportándose una causa de deceso que está relacionada con un estado inflamatorio agudo, shock hipovolémico y fracaso sistémico especial. Observando con atención los resultados que se reportaron para el laboratorio, el efecto de las lesiones pulmonares sobre el paciente pudo haber influido lo suficiente para poder ser incompatible con la vida.

Así como se mencionó anteriormente, condiciones precarias de alimentación y abandono, seguramente contribuyeron con la infección e hicieron que su desenlace fuese más temprano. Por tal motivo, los autores proponen realizar investigaciones in vitro con estos agentes emergentes, con el fin de generar una comprensión más amplia sobre su fisiopatología, ya que su distribución cosmopolita favorece la aparición de Histoplasma sp en lugares endémicos.

Se concluye que las enfermedades emergentes representan siempre un desafío a nivel veterinario, tanto en el diagnóstico como en el tratamiento, por lo cual es necesario establecer nuevas investigaciones que permitan mejorar el conocimiento de estas enfermedades, a efectos de poder garantizar la mejoría del pronóstico y tratamiento. 


\section{REFERENCIAS}

1. Brömel C, Sykes J. 2005. Histoplasmosis in dogs and cats. Clin Techn Small Anim Pract 20: 227-232.

2. Cordeiro, $\mathbf{R}$ et al. 2011. Serological evidence of Histoplasma capsulatum infection among dogs with leishmaniasis in Brazil. Acta Trop 119: 203-205.

3. Greenacre C, Dowling M, Nobrega LM. 2019. Diagnosis and treatment of histoplasmosis in a group of four domestic ferrets (Mustela putorius) and a review of histoplasmosis. J Exot Pet Med 29: 194-201.

4. Herraez P, Rees C, Dunstan R. 2001. Invasive fhaeohyphomycosis caused by curvularia species in a dog. Vet Pathol 38: 456-459.
5. Kenneth J, Ryan C, Ray G. 2017. Sherris: Microbiologia medica, $6^{\text {th }}$ ed., McGraw- Hil, Mexico, p. 670-674.

6. Klang A, Loncaric I, Spergser J, Eigelsreiter S, Weissenböck H. 2013. Disseminated histoplasmosis in a domestic cat imported from the USA to Austria. Med Mycol Case Rep 2: 108-112.

7. Larsuprom L. et al. 2017. Feline cutaneous histoplasmosis: The first case report from Thailand. Med Mycol Case Rep 18: 28-30 